\title{
Elbow-Anchored Interaction: Designing Restful Mid-Air Input
}

\author{
Rafael Veras \\ HMI Lab, Huawei \\ Markham, Ontario, Canada \\ rafael.veras@huawei.com \\ Ritesh Udhani \\ Dept. of Computer Science, \\ University of Manitoba \\ Winnipeg, Manitoba, Canada \\ udhanir@myumanitoba.ca
}

\author{
Gaganpreet Singh \\ HMI Lab, Huawei \\ Markham, Ontario, Canada \\ gaganpreet.singh2@huawei.com \\ Parth Pradeep Patekar \\ Dept. of Computer Science, \\ University of Manitoba \\ Winnipeg, Manitoba, Canada \\ parth.15je001620@am.ism.ac.in
}

\author{
Farzin Farhadi-Niaki \\ HMI Lab, Huawei \\ Markham, Ontario, Canada \\ ffarh101@uottawa.ca \\ Wei Zhou \\ HMI Lab, Huawei \\ Markham, Ontario, Canada \\ wei.zhou1@huawei.com
}

Pourang Irani

Dept. of Computer Science,

University of Manitoba

HMI Lab, Huawei

irani@cs.umanitoba.ca
Wei Li

HMI Lab, Huawei

Markham, Ontario, Canada

wei.li.crc@huawei.com
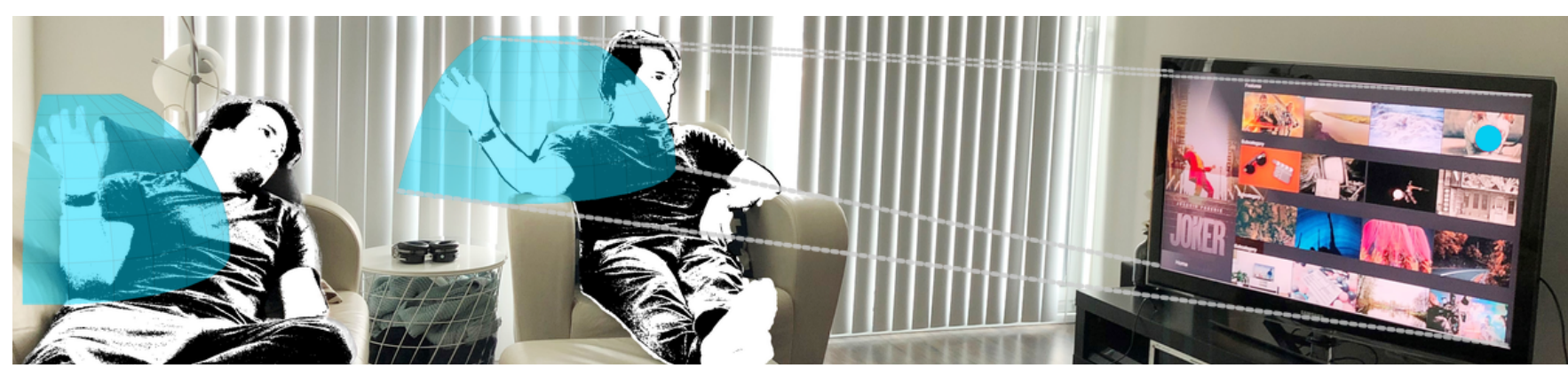

Figure 1: Elbow-Anchored facilitates casual seated postures for mid-air input. We observed users primarily anchoring their elbow either on a fixed surface or close to their body when sitting or resting in a casual posture. Based on this observation, we systematically explore the arm bio-mechanics involved in such a motion. We design mappings from motor (blue region) to screen space, implement a self-contained solution using a smartwatch (user's band), and study the performance and comfort of elbow-anchored interactions.

\begin{abstract}
We designed a mid-air input space for restful interactions on the couch. We observed people gesturing in various postures on a couch and found that posture affects the choice of arm motions when no constraints are imposed by a system. Study participants that sat with the arm rested were more likely to use the forearm and wrist, as opposed to the whole arm. We investigate how a spherical input space, where forearm angles are mapped to screen coordinates, can facilitate restful mid-air input in multiple postures. We present

Permission to make digital or hard copies of all or part of this work for personal or classroom use is granted without fee provided that copies are not made or distributed for profit or commercial advantage and that copies bear this notice and the full citation on the first page. Copyrights for components of this work owned by others than the author(s) must be honored. Abstracting with credit is permitted. To copy otherwise, or republish, to post on servers or to redistribute to lists, requires prior specific permission and/or a fee. Request permissions from permissions@acm.org.

CHI '21, May 8-13, 2021, Yokohama, Japan

(c) 2021 Copyright held by the owner/author(s). Publication rights licensed to ACM. ACM ISBN 978-1-4503-8096-6/21/05 ..\$15.00

https://doi.org/10.1145/3411764.3445546
\end{abstract}

two controlled studies. In the first, we examine how a spherical space compares with a planar space in an elbow-anchored setup, with a shoulder-level input space as baseline. In the second, we examine the performance of a spherical input space in four common couch postures that set unique constraints to the arm. We observe that a spherical model that captures forearm movement facilitates comfortable input across different seated postures.

\section{CCS CONCEPTS}

- Human-centered computing $\rightarrow$ Gestural input.

\section{KEYWORDS}

Mid-air Gestures, Comfort, Restful Input, Elbow-anchored Input, Variable-posture Gestures, Mid-air Gesture Fatigue

\section{ACM Reference Format:}

Rafael Veras, Gaganpreet Singh, Farzin Farhadi-Niaki, Ritesh Udhani, Parth Pradeep Patekar, Wei Zhou, Pourang Irani, and Wei Li. 2021. Elbow-Anchored Interaction: Designing Restful Mid-Air Input. In CHI Conference on Human 
Factors in Computing Systems (CHI '21), May 8-13, 2021, Yokohama, Japan. ACM, New York, NY, USA, 15 pages. https://doi.org/10.1145/3411764.3445546

\section{INTRODUCTION}

Mid-air interaction is moving beyond the lab and becoming available to consumers in real-world settings through platforms such as SmartTVs [1], smartphones [3, 5], in-car control systems [2], and Virtual Reality (VR) headsets [4]. Over the years, HCI researchers have studied and proposed guidelines for a variety of mid-air interfaces including mid-air pointing [10, 38, 46], mid-air menus $[8,11,18,27]$ and mid-air text-entry [32]. However, in a lab setting, experiments often require users to assume a certain degree of physical and mental attention, such as sitting straight and facing the system, to suit the technical capabilities of mid-air tracking systems. This does not reflect how users could interact with such systems in casual and more relaxed settings, where they may assume postures different than those evaluated in the lab [16]. If mid-air gestural interactions are to be integrated into consumer products, designers need to also consider the ergonomics and bio-mechanics of muscle and joint movements under casual sitting conditions.

Prioritizing user comfort, we examine how people sit on a couch (from a Western perspective) in a simulated home environment. Under casual postures, we ask users to emulate mid-air interactions with a smartTV, from which we identify their most common arm motions. We observe a strong preference for using the forearm and upper arm for such gestures. We also observe that shoulder rotations along with elbow flexion-extension constitute the majority of the movements across all postures. We refer to these forearm motions as elbow-anchored motions. These observation align with users' natural tendency to attempt to reduce arm and shoulder fatigue [21], also termed as the "Gorilla Arm Effect". Given that anchoring the elbow constrains user motion [10,23], we systematically examine the profile of "Elbow-Anchored" movement to enable efficient casual mid-air input. To identify the suitable parameters for such an input space we rely on the precision of a motion-capture system, and devise methods to map the range of such motion from 3D motor space to interact with planar content in visual space.

Based on the bio-mechanical properties of the arm, we modeled elbow-anchored gestures using a spherical movement, centered at the elbow. Our first mapping ElBOw-SPHERE, uses the entire forearm range and maps the Elbow-Anchored motion onto a 2D surface using an equirectangular projection. The ELBOw-PLANE mapping takes a subset, namely an inscribed plane within the spherical rangeof-motion, and uses an orthogonal projection to make use of the largest possible space within this region. Using a target selection study, we observe that using such parameters to design elbowanchored techniques is as efficient as unconstrained mid-air input. However, as expected, the former significantly reduces fatigue in comparison to full mid-air interaction. From our results, we are also able to derive the regions of highest throughput. Unaware of prior work on mid-air input across varying seated postures, we implement a self-contained prototype on a smartwatch, and assess the resilience of our model for elbow-anchored input when assuming different postures when rested on a couch. We found that the ElBow-SPHERE model can be adapted to the different postures we studied, with small variations in throughput depending on the posture and the spatial region. We report on such a non-uniform throughput space to assist designers with the design of elbowanchored interactions.

Our main contributions include: 1) an understanding of users' casual postures for mid-air input, leading to the design of elbowanchored interactions; 2 ) a systematic exploration of the necessary design parameters for enabling efficient elbow-anchored input; 3 ) an examination of our movement model's throughput and induced fatigue, in comparison to full arm mid-air input; and, 4) an investigation of our model's adaptability across different seated postures.

\section{RELATED WORK}

Our work lies at the intersection of identifying suitable mappings from mid-air input space to visual interfaces, and how these are affected by the ergonomics of constraining the upper limb motions which result when the user is sitting casually.

\subsection{Mid-Air Input Space and Mappings}

A key aspect of designing mid-air input concerns the mapping function for translating the hand motion to the cursor position on the screen. Myer et al. [37] suggest that directly mapping (ray-casting) hand motion provides an easy association for the user to keep track of the cursor. However, it tends to be imprecise. Similar findings were suggested by Vogel et al. [46] in their comparison of absolute, relative, and hybrid mappings of hand movements. The absolute mapping affords efficient input, at the cost of being erroneous, making it impractical. Cockburn et al. [14] also compared ray-casting with large $2 \mathrm{D}$ movements and movements in $3 \mathrm{D}$ volume. The $2 \mathrm{D}$ movements tend to be more precise and accurate than raycasting. $3 \mathrm{D}$ volume mappings are slower, less accurate, and more physically demanding than others. Solutions to implement natural but precise mid-air pointing include using a target-oriented approach $[9,13]$, velocity-oriented approach [33], or by allowing the user to manually switch between the absolute and relative mapping modes [15] which require explicit mode switching to mitigate erroneous input.

The literature also presents approaches for 2D plane mappings. Chattopadhyay and Bolchini [12] tested a menu based on directional movements (360 degrees) that required XY-plane movements of up to $18.9 \mathrm{cms}$, with a starting position at about shoulder level. The mid-air keyboard, Vulture [32], was implemented as a plane sized $20 \times 5.5 \mathrm{~cm}$. Cockburn et al. [14] used a square with sides of one cubit (a measure based on forearm length, $45.72 \mathrm{cms}$ ).

Previous work has also examined, to a minimal extent, spherical 3D input spaces. For instance, joint-centered kinespheres [31] are spherical 3D input spaces centered at joints. They tested three joints as the center of kinespheres: shoulder, elbow, and wrist, and found that wrist offers the highest performance (throughput) and comfort, followed by elbow and shoulder. Guinness et al. [19] propose a spherical input space defined by the user. In longitudinal user tests against planar and hyperplanar spaces, they found no significant difference in performance, arguing that their LeapMotion implementation which, does not provide precise forearm angle estimation, may have contributed to the results. Their participants were seated on an office chair with their elbow rested on a desk. In our studies, we use a motion capture system and a more ecologically valid setup that includes a large display and couch. Both 


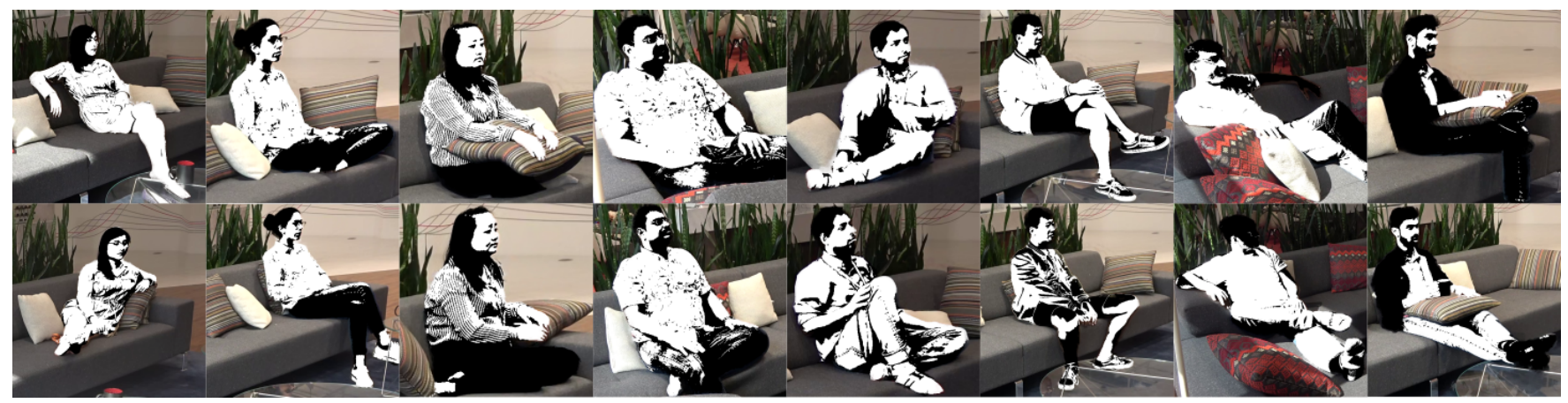

Figure 2: Body postures assumed by our participants in the observation study when asked to relax as if at home. Half the postures feature at least one elbow rested on a surface.

aforementioned works use the ISO 9241-9 "ring of circles" task, which consists of targets arranged as a ring around the center of the screen; as a result, the performance at the edges and corners of the input space was not examined. We consider this to be a significant gap, as biomechanical and environment constraints can impact performance unevenly in the space. In contrast, we devised a protocol for capturing performance measurements uniformly across the available motor-input space. Another example of spherical mapping, although not intended as a general-purpose input space, is Virtual Shelves [28] which projects a menu on a spherical space around the user. Similar to Virtual Shelves, TickTockRay [24] aims at providing a self-contained method for mid-air input in VR environments. But such an approach does not include the necessary parameters for casual elbow-anchored input.

In summary, researchers have studied a variety of methods that bound the 2D and 3D input spaces for mid-air interactions, but far less is known about how these apply to various casual postures.

\subsection{Biomechanics and Mid-air Ergonomics}

Mid-air interactions can benefit from an assessment of human biomechanical properties and upper limb ergonomics to offer an ideal user experience. König et al. [26] note the impact of hand tremors [45] on mid-air pointing imprecision. Temporal window averaging [37] or Kalman filter [42] techniques are needed to smoothen the pointing behavior. Another major issue with mid-air pointing is the "Gorilla Arm Syndrome" or arm fatigue [21]. Nancel et al. [39] investigated mid-air pan and zoom techniques for walldisplays and found that mid-air gestures are less efficient and more fatiguing than device-based gestures. Several methods have studied human fatigue in the context of mid-air input [21, 22, 29, 48]. As with these studies, we use the Borg CR10 tool in our evaluation of elbow-anchored interactions to assess the degree of fatigue induced by our approach in comparison to full-arm interactions.

Several studies suggest to limit the interaction time for which the arm/hand is raised in the air to mitigate fatigue effects [16, 23, 44] Hincapie-Ramos et al. [21] identified that movements further away from the user's waist will lead to higher fatigue and should be avoided. Liu et al. [30] explored arms-down positions with both hands at the sides of the body for interacting with a large display. Despite these suggestions, it is still unclear whether rested elbow interactions or interactions closer to the body can provide any significant performance benefits [10].

Nunnari et al. [41] observed subjects performing a 3D docking task while sitting on a chair and found that muscle load distribution is higher when subjects vary their posture. Their results suggest that postural variability may prevent over-exertion of key muscles, such as the shoulder, which is usually identified as the culprit for fatigue in mid-air gestural input [21]. In touch-based interaction, shoulder-dominant arm strokes are also known to require more muscle effort than their elbow counterparts, although with improved performance in target selection tasks [7]. Gesture execution and movements tend to change over the duration of an interaction, as individuals relax and adopt more comfortable positions [16]. However, certain postures encumber gesture execution. For instance, the degrees-of-freedom of hand trajectories are limited when the elbow is rested on a surface. Aslan et al. [6] also observed variation in gesture patterns. In the domain of virtual reality, Wentzel et al. [47] measured the maximum reach of an individual arm's and apply a non-linear transformation to extend user's virtual reach. Similarly, Ergo-O [34] decouples the physical and visual spaces and shrinks the physical space so that more objects lie within user's reach.

The above studies point at the need to consider system ergonomics and upper limb biomechanics to facilitate restful mid-air interactions. However, very little is known about the specific parameters needed to develop such interactions or how the constraints of a restful posture affect mid-air input. In particular, it is unclear if any advantage gained from diminished fatigue also negatively impacts the performance of such type of mid-air input.

\section{STUDY 1: CASUAL ARM MOVEMENTS}

The primary aim of this study was to analyse joint motions for the preferred arm movements during casual mid-air interactions. We presume that such seated postures would be selected by users to maximize comfort, a critical criteria when lounging on a couch, at home, for example. We restrict this exploration to postures on a couch as it is one of the more commonly available furnishings available in homes (in Western cultures), but also allows one to assume a number of restful postures. 


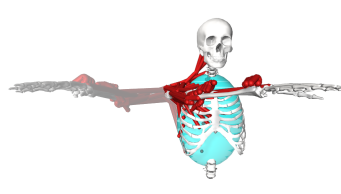

Shoulder Horizontal Adduction/Abduction

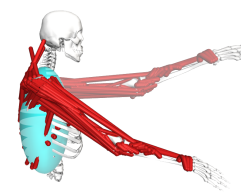

Shoulder Flexion/Extension

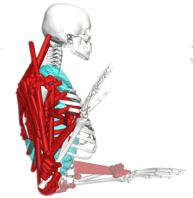

Elbow Flexion/Extension

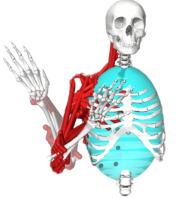

Shoulder Medial/Lateral Rotation

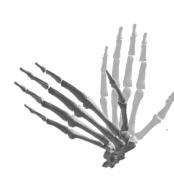

Wrist Deviation

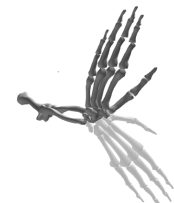

Wrist

Flexion

Figure 3: Upper limb joint motions used in coding our observational videos.

\subsection{Participants and Procedure}

Eight (8) participants, including 3 females, from our organization were invited into a space resembling a common living room and which included a large couch and TV. We instructed participants to sit on the couch in their most comfortable posture and perform a set of three different gestures: Swipe, Wave, and Slap. These three gesture labels were selected as they involve directional properties and can be distinguished from one another. The instructions on these three gestures were kept vague intentionally to support variability. We avoided using labels solely associated with any UI interactions such as drag, select, or move to prevent legacy bias [36]. For example, Swipe has a digital counterpart, but Slap and Wave gestures do not. While we acknowledge that the meaning of the term "comfort" may be subjectively distinct to each participant, we repeatedly asked participants to be relaxed and to assume they were in their own living room. Each participant performed Swipe, Wave and Slap gestures 3 times, in four directions (up, down, left, right) and with each hand. Variation in direction is important because the interaction of the body with furniture poses constraints to movement that are potentially asymmetric. These were captured in two of their most preferred body postures. The participants' body postures varied from sitting with their legs on the couch, resting their body on an arm rest, resting their arm on the back rest or on a cushion, among other postures they deemed to be comfortable (Figure 2). Interestingly, none of the participants chose to lie down. This could possibly be because the participants were not comfortable lying down in a professional environment.

We videotaped the sessions and recorded participants' comments. Sessions on average lasted an hour long.

\subsection{Movement analysis}

Two authors independently annotated each of the performed gestures based on the frequency of upper limb joints using Anvil [25], a video annotation tool. Video annotations were grouped into seven categories: motion joints, elbow position while rested, elbow position at the start of gesture execution, posture, gesture, gender, and hand. We define rested position as the neutral position of the joints when the participant is not doing a gesture and starting position as the position of the joints just before carrying out the gesture.

First, each gesture is characterized in terms of joint motions that are utilized in the movement. The movements are characterised by six joint motions [40]: shoulder horizontal abduction/adduction, shoulder flexion/extension, elbow flexion/extension, shoulder medial/lateral rotation, wrist flexion/extension, wrist deviation (Figure
3). One gesture can have annotations corresponding to multiple joint motions. For example, a swipe-left motion with the right hand may involve shoulder horizontal abduction/adduction, shoulder medial/lateral rotation as well as elbow flexion/extension. In the majority of cases participants brought their hand to a common starting position that was usually higher than the resting position. Since this motion is meant as an accomodation, and is not part of the gesture semantics, we did not count it.

The joint motions are associated with different upper limb segments. Combined, wrist deviation and wrist flexion/extension move the hand, shoulder medial/lateral rotation and elbow flexion/extension has an effect on the forearm, and finally shoulder flexion/extension and shoulder horizontal abduction/adduction move the upper arm [40]. Figure 3 depicts these joint motions and their effect on the upper limb segments.

\subsection{Results}

In Figure 4 we present a summary of the distribution of the joint motions for participants in each of the two postures in our study. We observed that the majority of movements involved participants lifting their elbow in order to bring the hand to a higher initial position for gesture execution. Those who anchored the elbow were either seated with the elbow rested on the arm rest, for example, or were interested in executing the gestures without affecting their seated posture.

We also observe that full arm motions are dominant in our observations, followed by forearm and hand movements (Figure 5-top). We note that a full arm motion is the one where we observed a major movement of the shoulder, while the other joints may also be moving simultaneously. Movements of the wrist were particularly high for the 'Wave' gesture, which dominated the counts presented in Figure 5-bottom. Users interpreted the 'Wave' motion in their actions literally.

We further examine joint motions across each of the postures assumed by the participants. We observed that vertical movements usually involved shoulder flexion/extension, which is a relatively strenuous joint motion. However, whenever the arm was rested at a high surface (e.g., backrest, armrest) shoulder medial/lateral rotation, a less demanding motion, prevailed. We also find that across all postures, elbow flexion-extension was involved, as well as shoulder medial/lateral rotation in all but one user posture. Horizontal movement was dominated by shoulder medial/lateral rotation for a majority of the participants. Some male participants also engaged in high-amplitude shoulder horizontal abduction/adduction, which 

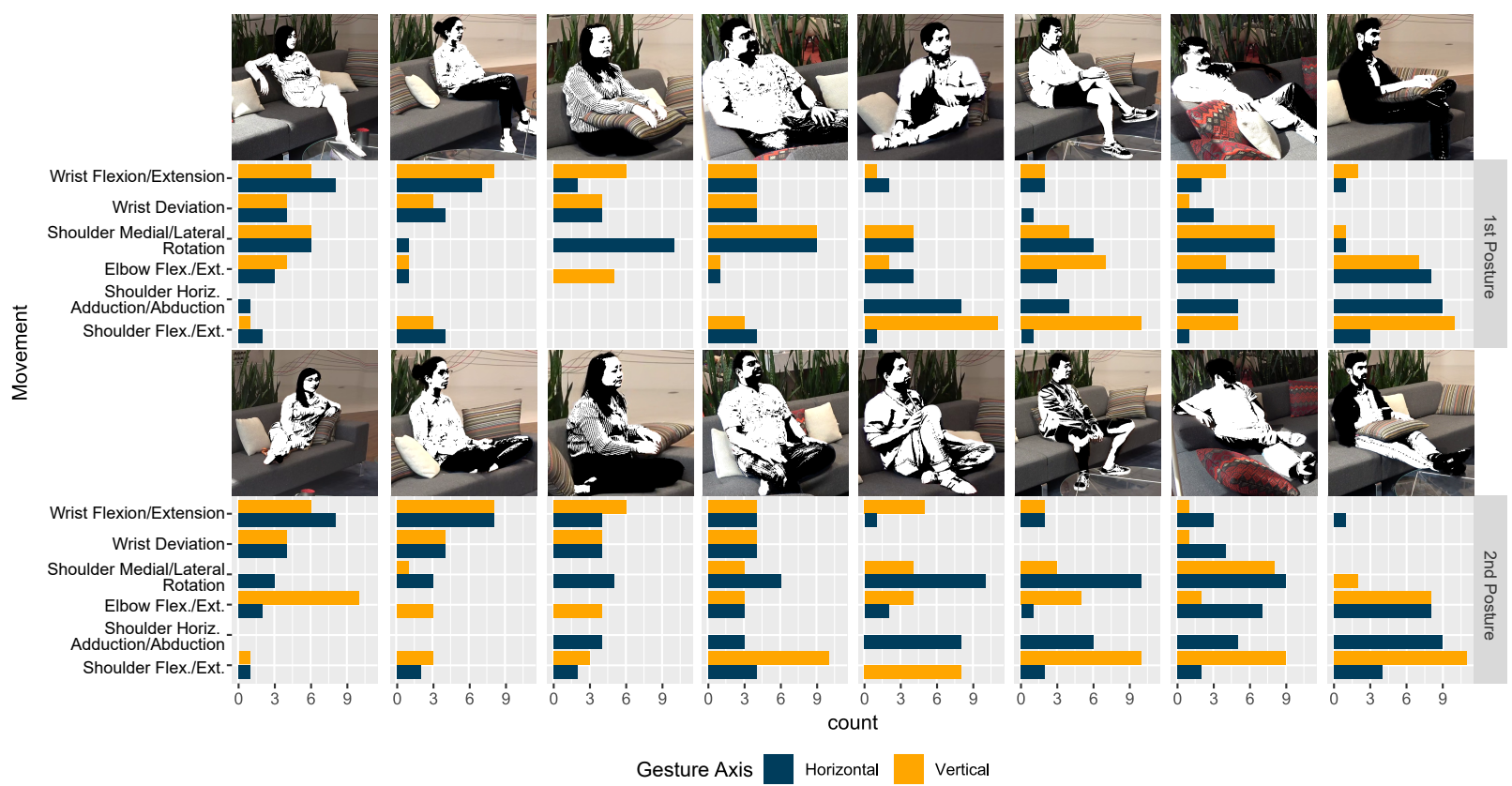

Figure 4: Frequency of all joint motions according to each of two postures assumed by participants. Horizontal gestures are those that imply lateral limb motion, such as "swipe left". Vertical gestures imply vertical limb motion, as in "swipe up".

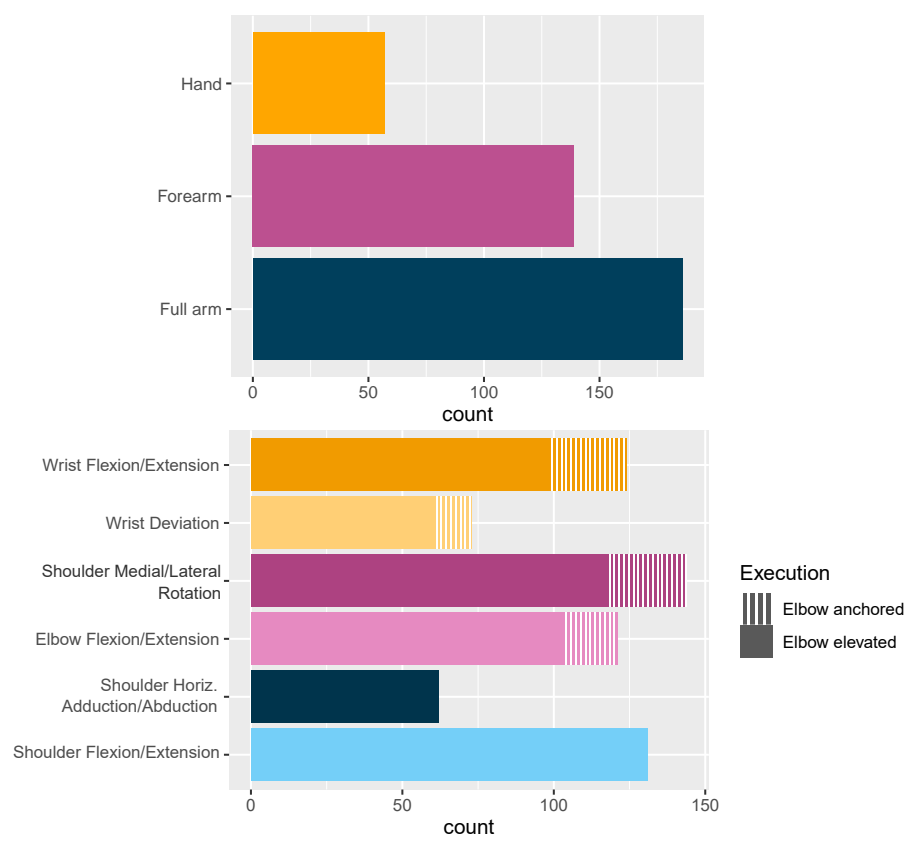

Figure 5: Frequency breakdown by limb segment(s) (top), and by joints (bottom).

is associated with high fatigue. Unlike female participants, who executed wrist motions more often than full arm movements.

We summarize our findings as a set of general observations from this study:
- The majority of upper limb motions involved the full arm and the forearm. The hand was primarily used in the 'Wave' gesture, but otherwise mostly accompanied the above two actions.

- When movement was annotated on joint, shoulder medial/lateral rotation (forearm) and shoulder flexion/extension (upper arm) were the most frequent.

- All users engaged in shoulder medial/lateral rotation (forearm movement) at least once (or more) across all postures, making it a common motion to explore.

- Male participants engaged in full arm motions whereas female participants executed smaller arm movements.

- In the vertical direction, users primarily engaged in shoulder flexion/extension (upper arm), while in the horizontal direction, users engaged in shoulder medial/lateral rotation (forearm).

- Posture alone is not a sufficient predictor for arm motion given a specific gesture. We observed variability in arm motion across participants that chose similar postures, which suggests personal preference is a major factor.

In contrast to full arm motions, we define elbow-anchored motions as those primarily involving the forearm (shoulder medial/lateral rotation and elbow flexion/extension) as seen from this study. We next explore the necessary parameters to characterize elbow-anchored motions (section 4 and section 5) and then investigate the throughput and induced fatigue for such type of mid-air input (section 6). Finally, we examine the potential for forearm motions across different seated postures. 

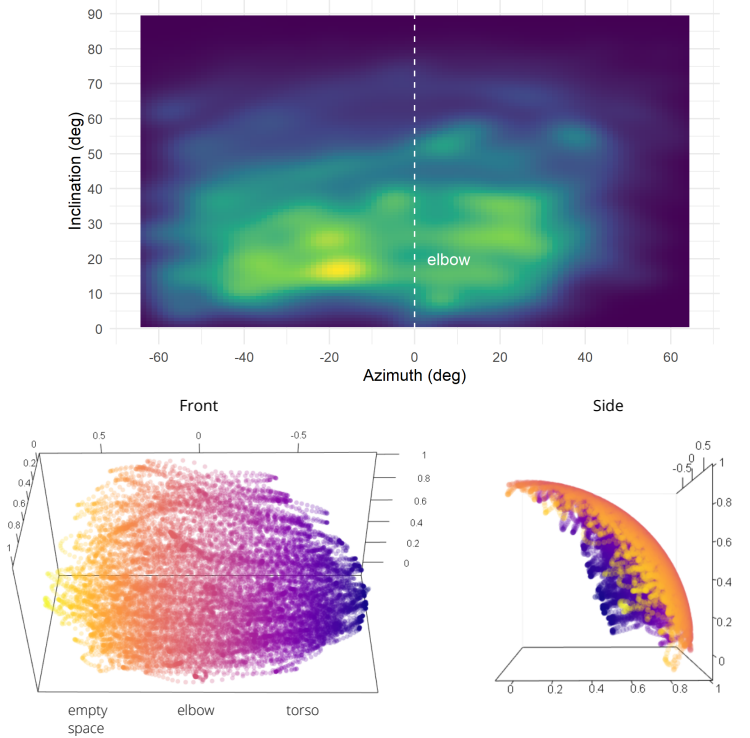

Figure 6: Top: Motion capture data showing the reachable hand positions when the elbow is anchored $(\mathrm{N}=8)$, spherical coordinates. The data was collected for the right hand, with the origin $(0,0)$ at the elbow location. Bottom: The same data in cartesian coordinates. The horizontal color gradient reveals the asymmetry of the reachable space, with a larger range of motion towards the body.

\section{OBSERVATION ON ARM RANGE OF MOTION}

The main objective of this exploration was to determine the fullcoverage of the volume reachable by the palm when primarily engaging the forearm. Eight participants (average age 31.87, 2 female) volunteered. We asked participants to repeatedly move the hand from left-to-right and right-to-left using their forearm all while gradually increasing the elbow flexion. We asked participants to do so until the top of the hand reaches the maximum flexion that could be comfortably achieved using the elbow. By "comfortable" we emphasized that participants should not overextend their arm beyond their natural limits. We also asked participants to anchor their elbow on the available armrest to obtain the most accurate movements with an origin at the elbow. Participants completed these movements on average within 3 minutes.

We used an OptiTrack motion capture system to record participants' forearm movements by placing the tracking markers on the elbow and at the back of the hand. These repetitive in-air movements produced a forearm reachable space when the elbow is anchored as shown in Figure 6.

The $2 \mathrm{D}$ projection represents the reachable space around the elbow joint if rendered directly onto a 2D display panel. It resembles an asymmetric oval region on the surface of the sphere. The forearm moves inward, towards the body, by at least $20 \%$ more than its movement outwards, away from the body. It also indicates a smaller movement space at higher levels of elbow flexion, and vice versa.
The lateral interaction space corresponding to a flexion angle of $0^{\circ}$ is $40 \%$ more than the interaction space at a flexion angle of $120^{\circ}$.

\section{MAPPING MOTOR TO VISUAL SPACE}

Based on the range of motion data we captured, we first define our $3 \mathrm{D}$ motor space, then project such a space onto the $2 \mathrm{D}$ visual space of a display.

\subsection{Defining the 3D Input Space}

Having established that the upper arm movement is more physically demanding than the forearm movement, we focus our design on the latter. Since the distance between the hand and the elbow joint is always the length of the forearm, the hand motion is defined by the sphere centered at the elbow joint $\mathrm{O}$ with radius the forearm (Figure 7).

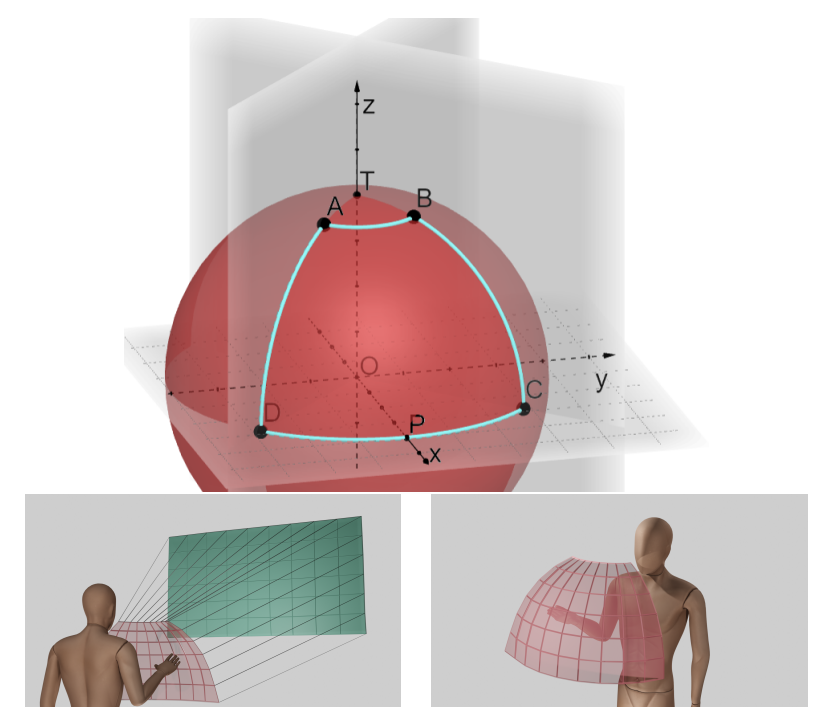

Figure 7: The motor space is defined as a subregion on a sphere, with the elbow at the sphere's center (top). Only a subsection of the sphere is accessible due to the range of motion of the elbow. The mapping of a spherical region to a $2 \mathrm{D}$ surface produces distortion (bottom). Regions in the top of the screen are mapped to smaller areas of the input space. In practice, this leads to a variable control-display ratio.

Not everywhere on the sphere surface is reachable by the hand. To describe regions on the sphere that are reachable (blue outline in Figure 7), we consider four planes: $O T A D, O T B C, O D P C$, and the plane that crosses $A$ and $B$ and is perpendicular to $\mathrm{z}$. Without loss of generality, assuming the user is right handed, the plane OTAD corresponds to the rightmost limit the user is able to move the hand to, and the plane $O T B C$ corresponds to leftmost limit the user is able to move the hand to. We observed that our study participants performed gestures with the hand raised above a certain level, which we approximate by the plane ODPC for consistency and simplicity. On a couch this plane may represent the level of armrest.

In the previous section, we estimated the maximum angles for a person sitting straight and anchoring the elbow on an armrest. 
In this setting, the maximum angle range $\angle D O C$ of the left-right forearm movement is around $100^{\circ}$, with points $C$ and $D$ being the bottom left and bottom right corners of the input space. The angle range $\angle D O A$, which is same as $\angle C O B$, for the up-down movement is around $70^{\circ}$. The points $A$ and $B$ are, respectively, the top right and top left corners of the reachable space. We set a coordinate system that aligns the $\mathrm{x}$-axis with $O P$ and z-axis with $O T$.

As an example, we have angles $\angle P O D=-60^{\circ}, \angle P O C=40^{\circ}$, and $\angle D O A=\angle C O B=70^{\circ}$. This allows any point in the input space to be specified by two angles $\theta \in\left[-60^{\circ}, 40^{\circ}\right]$, and $\phi \in\left[0^{\circ}, 70^{\circ}\right]$, which we refer to as azimuth and inclination, analogous to the longitude-latitude geographic coordinate system on Earth.

\subsection{Input-Output Mapping}

After defining the spherical input space, we need to define a mapping to the output space, which in this paper corresponds to a large $2 \mathrm{D}$ display. We know it is not possible to project a 3-dimensional spherical surface to a 2-dimensional display while perfectly preserving areas and shapes. This is akin to the problem of map projection. For example, the shape or the size of the polar regions of Earth are typically severely distorted in a 2D map comparing to that of the tropical regions. There are different ways to map the angles $(\theta, \phi)$ to the display coordinates. We consider a linear mapping between spherical and Cartesian coordinates:

$$
\theta=\arctan \frac{y}{x} \quad \phi=\arccos \frac{z}{r}
$$

where $\theta$ is the azimuth, $\phi$ is the inclination angle, and $r$ is the length of the forearm. Figure 7, bottom, depicts how input space regions are mapped to display regions. Note that distortion increases with inclination angle, with the top part of the screen being mapped to a small area of the spherical surface. As a result, the spherical input space has a variable control-display ratio, which would manifest as the cursor moving at faster in the top than in the bottom. This could affect motor control and the precision of target selection. In order to examine these issues, we present a controlled experiment in the next section.

\section{STUDY 2: INPUT SPACE FATIGUE AND THROUGHPUT}

We designed a controlled experiment to assess the effect of input space on target selection performance and fatigue. In particular, we examine the position and geometry of input spaces. We compared two input spaces anchored to the elbow with an input space anchored to the shoulder. At elbow level we tested two mapping functions, which correspond to different input shape geometries: plane and sphere.

\subsection{Experiment Design}

We use a single-factor, within-subjects design. Each participant experienced three types of input spaces: ELBOW-SPHERE, ELBOWPLANE, and SHOULDER-PLANE. To collect meaningful fatigue data, we controlled the duration of a session instead of the number of targets. As a result, the number of targets selected in a session varied with participant performance; likewise, the number of observations for targets at the end of the study is not uniform. The input spaces are as follows:
6.1.1 Elbow-Sphere. The spherical input space proposed in this paper, where forearm inclination and azimuth angles are mapped to the screen $y$ and $x$ coordination, respectively. The origin for calculation of these angles was the elbow position at the armrest. The boundaries of the input space were defined per-individual, according to a calibration procedure detailed in the next section.

6.1.2 Elbow-Plane. A plane parallel to the screen and orthogonal to the armrest is positioned so that its bottom edge is at armrest level. In a Y-up, Z-forward coordinate system, the $x$ and $y$ coordinates of the hand are mapped to the screen coordinates, while the $z$ coordinate of the hand is ignored. This input space was scaled according to the spherical boundaries resulting from our calibration step, as in Figure 8. Scaling ensures that the relation between the dimensions of the spaces under comparison remains constant across participants.

6.1.3 Shoulder-Plane. Identical to ELBow-PLANE, with the exception of a vertical offset of 1 forearm length, which positions the plane at roughly shoulder level.

\subsection{Tasks and Measures}

Participants were exposed to a target selection task based on Fitt's Law. Participants' movements were mapped to a green circular cursor. The targets, also circular, were displayed one-at-a-time and were white on black background; they turned red when the center of the cursor was within their boundaries. The index of difficulty (ID) of a target was randomly sampled from six preset values (from 1 to 5). Given an ID and the position of the previous target, the target position was determined randomly. Target size (circle diameter) was determined according to the Fitt's Law formula:

$$
\text { ID }=\log _{2}\left(\frac{D}{W}+1\right)
$$

This generation process was run once, and produced a single list of targets that were used across participants and conditions. We recorded (a) movement time as the time elapsed from the previous selection to the next, (b) correctness as to whether or not the center of the cursor was within the target at selection time, and (c) the BORG fatigue level at 30s intervals. Order was counterbalanced.

\subsection{Procedure and Apparatus}

Participants were seated on a lounge chair positioned two meters away from a 85 " TV. They were instructed to seat comfortably and place their dominant arm on the armrest. Participants' movements were tracked with 10 Optitrack $17 \mathrm{~W}$ cameras. We attached a small circular rigid body directly to elbow and large rigid body to the wrist with a glove.

Before starting any tasks, we performed a calibration step to determine the range of motion of the participant's forearm in the rested position. During calibration, the maximum angles for the spherical input space were progressively reduced so that an onscreen cursor controlled by the participant could reach the four corners of the TV. We did not make a judgement of the difficulty of reaching these boundaries, as this information will be present in the selection data; as such, we merely ensured that the boundaries of the output space were reachable. 


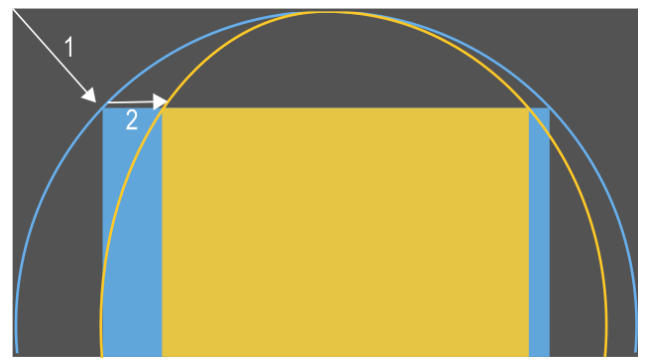

Figure 8: Procedure for scaling planar input spaces. We begin with the gray plane that encloses a full $[-90,90]$ spherical input space (blue). This plane is then scaled (1) to fit within the spherical region, while maintaining its aspect ratio. Given arbitrary spherical boundaries (yellow), we further reduce the planar boundaries (2), now with free aspect ratio.

Participants were introduced to the task and presented with practice runs before each set of trials (one set per condition). They practiced until they felt ready to begin the task. For each condition, participants were asked to select circular targets on-screen for 5 minutes or until they were too tired to continue. This led to some variability in the number of observations per participant but did not affect our analysis as it does not require balanced samples. At 30-second intervals, participants were prompted to rate their level of arm fatigue on a BORG scale. We delayed the BORG scale appearance until selection of the current target was completed, so in practice, the length of this interval varied slightly. The 5-minute threshold did not include the time taken to periodically rate arm fatigue. Participants were asked to rest as much as possible between each set of trials.

Selection was done with the non-dominant hand by pressing the buttons of a bluetooth mouse. While not realistic, this choice of selection adds minimal overhead, allowing us to clearly measure the effect of movement. This is important because we are interested in the relative differences between the input spaces rather than in a real-world measure of performance.

\subsection{Participants}

We recruited 9 participants (5 males) within our organization, aged 22 to 35 years old. All participants used their dominant hand.

\subsection{Results}

Following, we present statistics and significance tests for fatigue, movement time, and throughput. We are interested in the effect of input space on these variables. We fit linear mixed models with random intercepts for participant, and test significance with Wald $\mathrm{t}$-tests on the null hypothesis that the effect estimates are zero. We report coefficient estimates as $\beta$, along with their standard error, as well as $\mathrm{t}$-values and $\mathrm{p}$-values. Models were fit using the $\mathrm{R}$ package nlme [43]. In total, we collected 3,332 target selection observations from 9 participants.

6.5.1 Fatigue. Figure 9 shows a pronounced difference between the fatigue curves of the elbow-anchored input spaces and that of

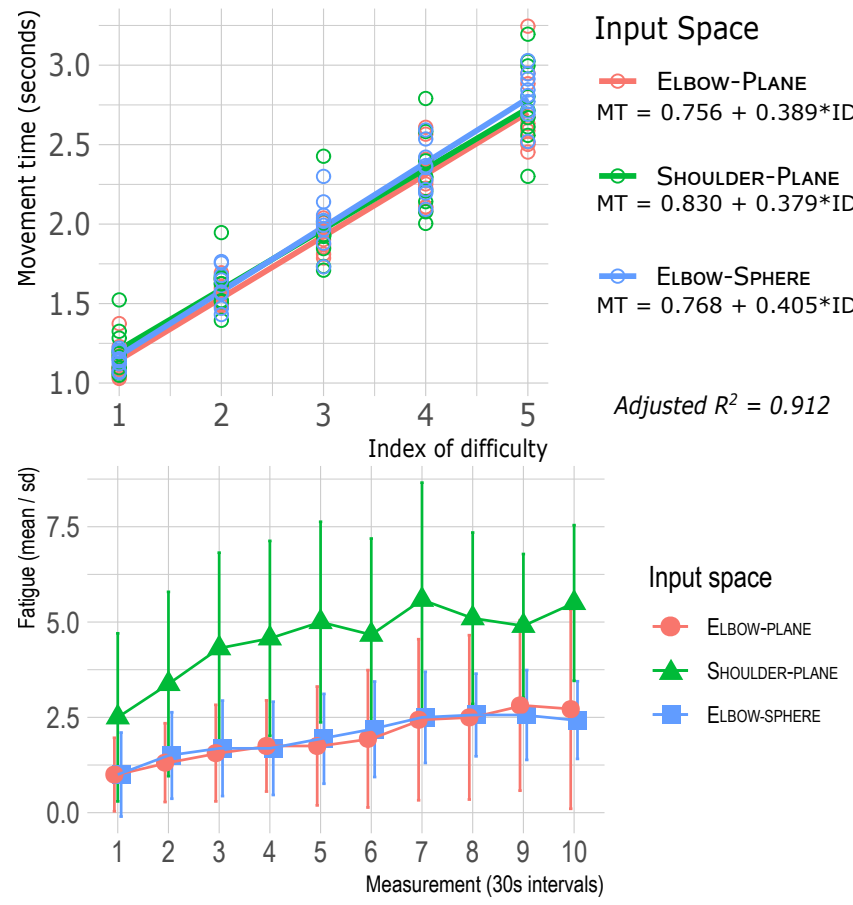

Figure 9: Top: linear models fit to participant means. Bottom: mean and standard deviation of fatigue measurements (BORG scale) taken over a period of 5 minutes at 30s intervals.

SHOULDER-PLANE. For all nine participants, the maximum level of fatigue was experienced in the SHOULDER-PLANE condition. 4 out of 9 participants withdrew the SHOULDER-PLANE block before having completed 5 minutes of task time due to extreme arm fatigue. We tested the significance of fatigue differences by fitting a linear model to the normalized BORG data, with input space (dummy-coded) and time as predictors. Table 1 features the parameters of this model, with SHOULDER-PLANE as the baseline. The statistically significant coefficients for ELBOW-PLANE and ELBOW-SPHERE confirm that the SHOUlDER-PLANE input space is indeed more tiring. The interaction between input space and the effect of time was not significant.

Table 1: Linear model fit to fatigue data with baseline set to SHOULDER-PLANE. The variable time is ordinal. The model reveals that elbow-anchored input spaces accounted for a statistically significant $(p<.05)$ reduction in fatigue.

\begin{tabular}{rrrrr}
\hline & & Std. & & \\
& Estimate & Error & t value & p value \\
\hline (Intercept) & 2.975 & 0.441 & 6.745 & 0.00 \\
ElBOw-PLANE & -2.097 & 0.610 & -3.435 & 0.00 \\
ELBOw-SPHERE & -1.890 & 0.610 & -3.096 & 0.00 \\
time & 0.292 & 0.078 & 3.728 & 0.00 \\
Interaction (w/ time) & & & \\
ElBOw-PLANE & -0.091 & 0.104 & -0.877 & 0.38 \\
ELBOw-SpHERE & -0.124 & 0.104 & -1.187 & 0.24 \\
\hline
\end{tabular}


6.5.2 Movement Time. We fit a linear model to the data with input space and ID as predictors of movement time, including a random intercept for the variable subject (Figure 9). In this model, $R^{2}$ (adjusted) $=0.5367$, the effect of input space was not significant: $\beta($ ShOUldER-PLANE $)=0.055, \mathrm{SE}=0.05, t_{3318}=1.09, p=0.274$, and $\beta$ (Elbow-SPHeRe $)=0.019, \mathrm{SE}=0.05, t_{3318}=0.40, p=0.686$.

6.5.3 Effective Throughput. Effective throughput is a performance measure that accounts for the time-accuracy trade-off and computed as:

$$
T_{e}=\left(\frac{I D_{e}}{M T}\right),
$$

where $I D_{e}$ is the Index of Difficulty calculated with the effective width $W_{e}$. For each of our circular targets, $W_{e}=4.133 \times S D_{d}$, where $d$ is the Euclidean distance between the observed selection and the previous selection (amplitude). $I D_{e}$ is computed for each target with data from all participants. Figure 10 shows the throughput means and $95 \%$ confidence intervals computed over the participant means for each input space condition. These measures were calculated following the method of Morey [35] for within-subject designs. We tested the effect of input space on throughput with a repeatedmeasures ANOVA, and found no statistically significant differences $\left(F_{2,16}=0.663, p=0.529\right)$.

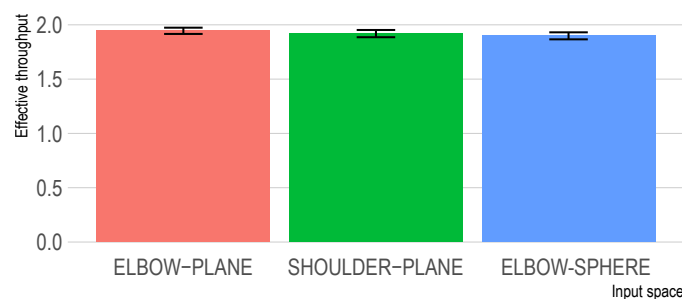

Figure 10: Means and 95\% confidence intervals for effective throughput.

6.5.4 2D Throughput. With participants seated comfortably on a lounge chair and having their arms rested, it is plausible that performance, as measured with throughput, is not distributed uniformly over the space. In order to examine this hypothesis, we fit linear models for each input space with throughput as a response variable and the $\mathrm{x}$ and $\mathrm{y}$ screen coordinates of the targets as predictors. We used these models to generate the fine-resolution heatmaps shown in Figure 11. The heatmaps reveal distinct patterns for each input space. In SHOULDER-PLANE, the top part of the space has worse performance, probably for requiring more physical effort to reach. With ElBow-PLANE the throughput gradient flows from left to right Notably, the spherical input space features a region with very low throughput in the top left corner, which indicates that participants had trouble reaching that region.

\subsection{Discussion}

Our results show a surprising lack of trade-off between performance and fatigue. The heightened fatigue of the SHOULDER-PLANE condition did not translate to worse or better performance. Despite ensuring that users would not get over-fatigued using pilot studies (to be compliant with our research ethics), fatigue was high enough in that condition to motivate four participants to quit before the end of the 5-minute session. We believe performance time would begin degrading after such a threshold. This outcome is also not uncommon as earlier work points at, under some instances, increased fatigue without degrading performance times [19, 21, 23]. The dimensions and position of Elbow-PLANE and Elbow-SPHERE are similar, but these spaces constitute very different interaction models, due to the non-linear nature of the control-device ratio in ELBOW-SPHERE; that is, regions at the top of the output space correspond to smaller regions of the input space. In practice, this leads to the cursor moving much faster in the top. Nevertheless, the overall performance of ELBOW-PLANE was not significantly different than that of ELBOw-SPHERE, which is consistent with previous findings [19]. Upon closer inspection we found that the performance of these spaces is distributed differently. In particular, we see a bottleneck in the top-left corner with ElBow-SPHERE. This issue can be attributed to a motor constraint: at high elevation angles, it is difficult to move the arm from right to left when the palm is facing the screen. While turning the hand sideways makes this movement easy, participants could have ignored this possibility under an assumption that the screen needs to see the palm.

The equivalent overall performance of ELBOw-SPHERE and ELBOwPLANE suggests that ELBOW-SPHERE compensates in other regions the poor of performance in the top-left corner, specially in the bottom-left corner. We consider this factor in our next study, exploring the impact of posture variances on anchored-elbow input.

\section{STUDY 3: SPHERICAL INPUT ACROSS DIFFERENT POSTURES ON THE COUCH}

Having established that a spherical input space anchored at a user's elbow potentially supports restful interaction in a variety of postures, we examine in this section the consistency of the interaction across postures. Are users able to maintain an acceptable level of performance and comfort in typical postures? Are there performance patterns that could motivate modifications of this input space? We present an exploratory user study where participants were asked to select mid-air targets while resting on a couch in various postures.

We only carry out this exploration with the ElBow-SPHERE mapping for two reasons. First, including the Elbow-PLANE and SHOUlDER-PLANE in study 3 would dilute our study goals. Furthermore, ELBOW-SPHERE can be implemented in a self-contained manner on a smartwatch, validating the potential for non-reliance on a global tracker and thus usable under various comfort postures.

\subsection{Apparatus and Materials}

The spherical input space can be implemented with any wearable device that provides a rotation vector, such as a smartwatch. By comparison, planar input spaces would require an additional piece of information, the forearm length, for conversion between spherical coordinates to Cartesian coordinates. We developed a smartwatch application on Android that allows mid-air control of a remote cursor. On the host computer, a client establishes a TCP channel with the watch. The client listens for packets containing real-time azimuth and inclination values, which are used to move the cursor. 

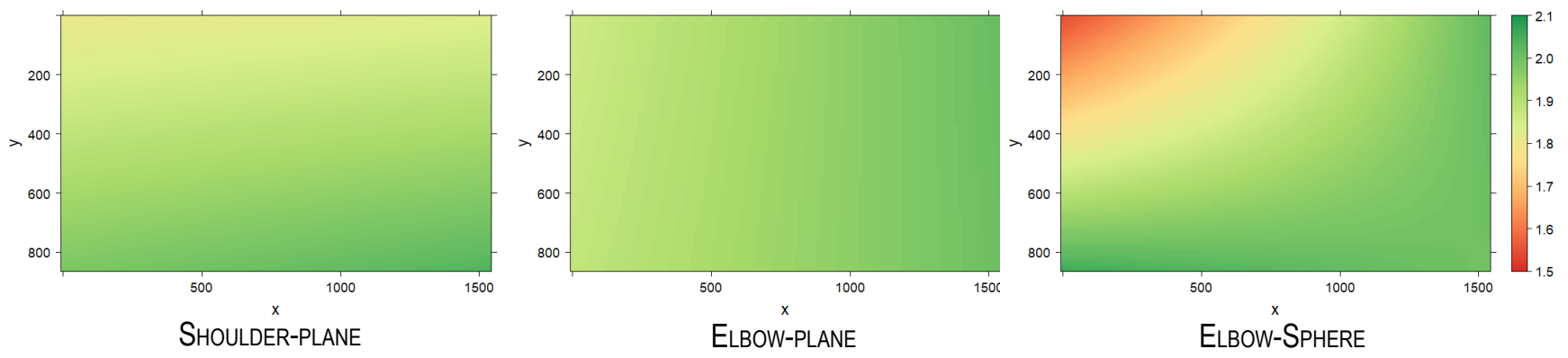

Figure 11: Multivariate linear models fit to the throughput data. $\mathrm{X}$ and $\mathrm{Y}$ screen coordinates are modelled as predictors for throughput.

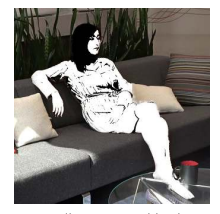

(1) Elbow rested high

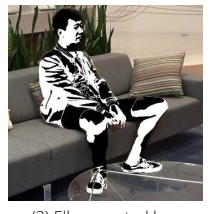

(2) Elbow rested low

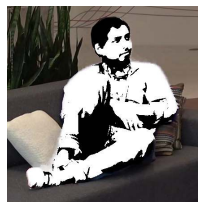

(3) Sideways seated

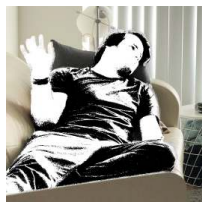

(4) Sideways lying
Figure 12: We tested the spherical input space in four postures.

On the smartwatch, the application is a Wear OS Watch Face, an always-on, readily accessible application responsible for displaying the clock. The app reads the Game Rotation Vector from the Android Sensors API. This vector contains rotation measurements relative to a fixed reference coordinate system that corresponds to the device's default orientation. With the smartwatch attached to the user's wrist, this vector gives us directly forearm azimuth and inclination; however, a calibration step is necessary to adjust the reference coordinate system. We added a calibration button to the watch face, which users press while holding the forearm at rested position, parallel to the ground and orthogonal to the torso. This method does not require the user to be facing the TV. For selection, we used mouse clicks with mouse controls in the hand opposite to the one with the smart watch, however, it could also be implemented using a rapid index to thumb pinch [17]. We used mouse device for selection in order to allow user to select even the smallest of the targets, measuring as small as 12 pixels, generated from higher difficulty levels. This prevents any possible confounding errors due to selections made from the same hand that is used for pointer control, thereby keeping the observations valid and also isolating the effect of movement from the selection method.

\subsection{Experimental Design}

We employ a target selection task to compare mid-air interaction under four body postures, using a spherical mapping between motor and display space. The study follows a $4 \times 8$ within-subject design, with posture and target position as factors. We define four index of difficulty (ID) levels $(2,3,4,5)$ and four postures (Figure 12):

- Elbow Rested High - Seated, elbow rested at shoulder level

- Elbow Rested Low - Seated, elbow rested at waist level
- Sideways Seated - Seated sideways (lengthwise), elbow rested at waist level

- Sideways Lying - Lying sideways

The postures are representative of most postures one can assume in a common couch and, with the exception of Sideways Lying, capture the patterns seen in our observational study. Each posture presents distinct motor constraints to the arm. People either sit or lie on the couch. When seated, they rest their feet on the floor or on the couch (in a sideways orientation relative to the display). In addition, people can rest their elbow on a lower surface (armrest or waist) or higher surface (backrest).

The height at which the elbow is rested influences the range of motion. With the elbow rested low, an upward motion is achieved via elbow flexion, while with the elbow rested high (e.g., on the backrest) the same motion is achieved via shoulder lateral rotation. Likewise, horizontal motion can be achieved through shoulder medial/lateral rotation or elbow flexion depending on where the elbow is rested. Furthermore, with the elbow rested low the body becomes an obstacle. In the sideways postures, the couch backrest becomes an obstacle.

\subsection{Task and Materials}

We used a Fitts Law-style target selection task where eight circular targets were arranged in a grid (Figure 13a). Each target was presented one at a time along with a reciprocal target (i.e. same size) located at the center of the screen (Figure 13b). The role of the reciprocal target was to establish a common origin for all target selections and to allow measurement of selection time for each target, uniformly. Each selection task started with a reciprocal target, highlighted through an accent filling color, to be selected. Once the reciprocal target was selected, the actual target to be selected next was highlighted and the user was required to make a selection again. This active state switching repeated 5 times for each target and until all targets were selected in an ID. We manipulated ID by varying target size. Note that targets with the same ID have different sizes if they are not equally distant from the center. As we collected 5 observations per target, a total of 640 observations per participant were generated ( 8 target positions $\times 5$ repetitions $\times 4$ ID $\times 4$ postures). 


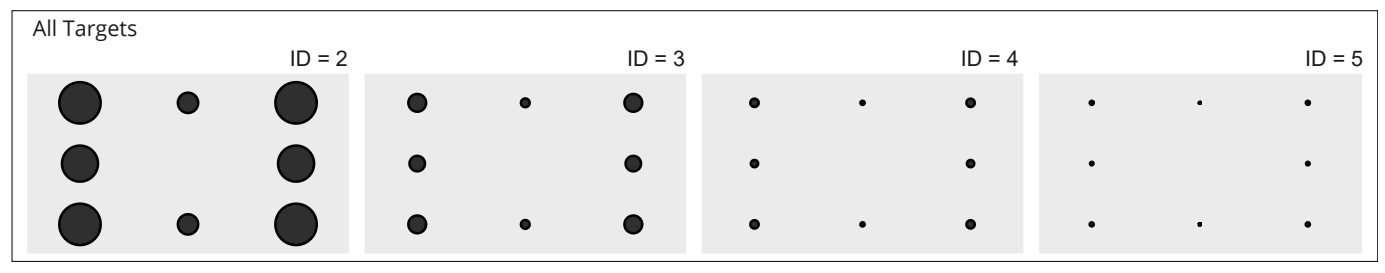

(a)

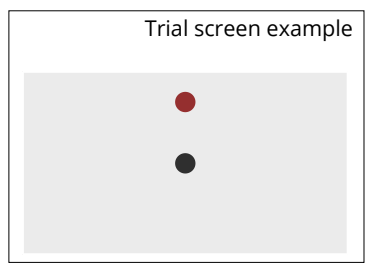

(b)

Figure 13: a) All targets used in our controlled study split by index of difficulty (ID = 2 to 5). b) Each target was presented along with a reciprocal positioned at the center of the screen. Participants selected each target five times.

\subsection{Procedure}

Participants were instructed to sit on the couch in the first posture assigned to them. The order of postures was counterbalanced using a Latin square design for each participant. The experiment facilitator described the calibration procedure and the task, then instructed participants to perform calibration followed by a practice run of the task. Participants were instructed to "select targets as fast as possible while keeping the accuracy at a constant level" [7] During the practice run, participants were instructed to recalibrate if necessary. This procedure was repeated for every posture. After the completion of the tasks for each posture, the facilitator administered the Raw NASA-TLX (RTLX) questionnaire [20] and enforced a 5-minute break. The tasks within a posture block were presented without interruptions, but participants were allowed to rest anytime. Upon completion of the whole experiment, participants filled a custom questionnaire.

\subsection{Results}

We ran the experiment with 8 participants ( 2 female) aged between 21 and 38 years old. We collected 5,120 observations (target selections) in sessions that averaged 1 hour and 46 minutes excluding the time needed for calibration. After removing outliers with the interquartile range method, 5,060 observations remained. We use repeated measures ANOVA for statistical tests, with Tukey's HSD for post-hoc analysis. Where appropriate, we plot means and 95\% confidence intervals calculated with Morey's [35] method for within-subject designs.

7.5.1 Posture and Target Position. The mean effective throughput was $1.88 \mathrm{bits} / \mathrm{s}$, calculated in the same way as study 2 . The frontal postures (1 and 2) had throughput slightly higher than average (1.92 and 1.89), while the lying posture had throughput slightly lower (1.84). The aggregate differences in performance by target position (Zone) were more pronounced. Notably, targets that required horizontal movements (West and East) had throughput much higher than targets that required vertical movements (North and South); for instance, West was 30\% higher than North (Figure 14).

Our tests revealed an interaction between Posture and Zone $\left(F_{21,5021}=6.46, p<.001\right)$, and post-hoc analysis pointed to statistically significant differences $(p<.005)$ between postures in all zones but $\mathrm{E}$ and $\mathrm{S}$ (Table 2). These differences range from -0.3 to $0.28 \mathrm{bit} / \mathrm{s}$. In the NW zone (top-left corner) the best performance was achieved with the sideways seated posture and the worst with elbow rested high. However, in both $\mathrm{N}$ and $\mathrm{W}$ zones performance with elbow rested high was significantly higher than with other postures. In zone SW (bottom-left corner), participants seated sideways achieved lower performance than under other postures. In zone NE, sideways lying had the worst throughput. And in zone SE (bottom-right corner) elbow rested low had the best throughput.

Table 2: Post-hoc pairwise comparisons with $p$-values adjusted with Tukey's HSD. The estimates are for the difference in mean throughput between postures within target zones. Only the statistically significant differences are displayed. For all rows, degree of freedom $=5021$.

\begin{tabular}{lrrrrr}
\hline & Posture & & & & \\
Zone & Diff. & Estimate & SE & t.ratio & p.value \\
\hline NW & $1-2$ & -0.252 & 0.059 & -4.30 & 0.0001 \\
& $1-3$ & -0.296 & 0.058 & -5.08 & $<.0001$ \\
& $2-4$ & 0.220 & 0.059 & 3.77 & 0.001 \\
& $3-4$ & 0.264 & 0.058 & 4.55 & $<.0001$ \\
N & $1-2$ & 0.231 & 0.059 & 3.93 & 0.0005 \\
& $1-4$ & 0.185 & 0.059 & 3.15 & 0.009 \\
& $2-3$ & -0.164 & 0.058 & -2.82 & 0.0249 \\
NE & $1-4$ & 0.282 & 0.058 & 4.85 & $<.0001$ \\
& $3-4$ & 0.172 & 0.058 & 2.94 & 0.0172 \\
\hline \hline & Posture & & & & \\
Zone & Diff. & Estimate & SE & t.ratio & p.value \\
\hline SE & $1-2$ & -0.155 & 0.058 & -2.67 & 0.0378 \\
& $2-4$ & 0.154 & 0.058 & 2.64 & 0.041 \\
SW & $1-3$ & 0.155 & 0.059 & 2.63 & 0.0431 \\
& $2-3$ & 0.233 & 0.059 & 3.93 & 0.0005 \\
W & $1-2$ & 0.281 & 0.059 & 4.77 & $<.0001$ \\
& $1-3$ & 0.234 & 0.059 & 4.01 & 0.0004 \\
& $1-4$ & 0.227 & 0.059 & 3.87 & 0.0006 \\
\hline
\end{tabular}

7.5.2 Questionnaires. The NASA TLX data (Figure 15, left) shows that the spherical interaction with elbow rested high was associated with higher physical demand, effort, and frustration, while interaction in the seated sideways posture had the lowest physical demand and effort. Curiously, the $95 \%$ confidence intervals show that participants' perception of their own performance in each postures was not systematically affected by their perception of effort. Our second questionnaire asked participants to provide two rankings of the postures: one where they weigh the postures according to 

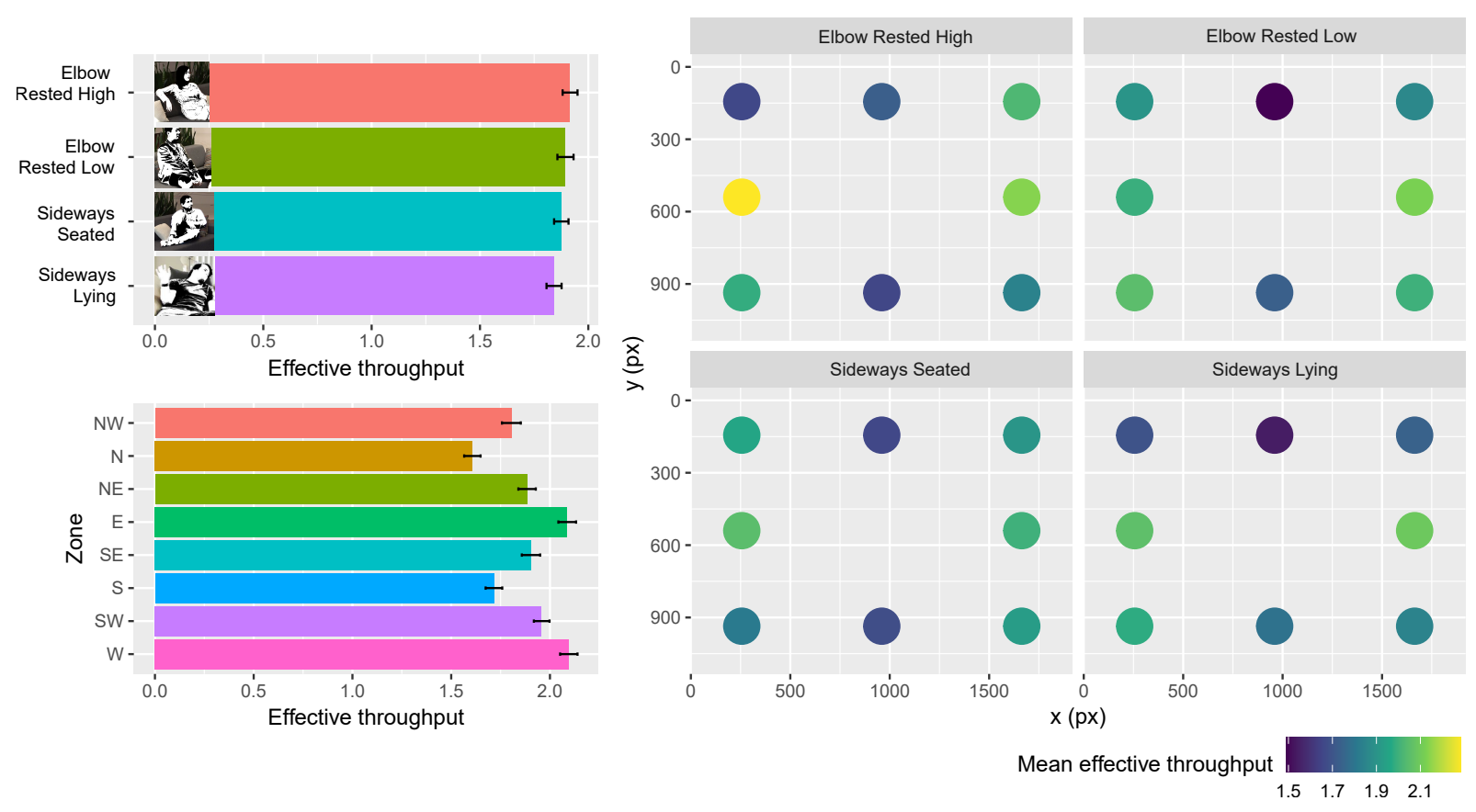

Figure 14: Effective throughput of postures and target zones (left), and effective throughput for target zones within each posture (right). We found a statistically significant interaction between target zone and posture.

their experience in the study, and another where they disregard the experience in the study. Generally, the results show that posture preference in the study was well aligned with participant's prior preferences. This is a positive result for the spherical interaction, as it did not seem to affect participants' postural preferences. Despite this general pattern, one participant stated that mid-air interaction would motivate them to change posture: "I do sit in what I ranked as 1 and 4 (when TV is in front of me) and 2 and find those comfortable but if I needed to use a smartwatch to control the screen I would sit up most likely and not be lying down." A second participant reported neck and shoulder discomfort in postures 1 and 4: "In general, the calibration and the cursor works well, although posture 1 (elbow rested low) and 4 (sideways lying) cause the tiredness for my neck and shoulder. The final level is difficult and tricky to do accurately". Only one participant reported not using any of the study postures routinely; most participants reported frequently being on the couch in at least one of the postures.

\subsection{Discussion}

Differences in performance due to postures were relatively small and dependent on target location. Our results point to difficulties with straight vertical movements towards the edges of the display. This problem may stem from a potential asymmetry in the calibration of input spaces; that is, the central axis of the space is not necessarily aligned with what participants identify as the central position of their forearm. Consider the case where the elbow is rested on the armrest of an office chair: a user may think that the center of the space is aligned with the armrest, but depending on the calibration, it may be offset towards the torso, since the accessible area in front of the body is larger than the area on the other side of the armrest. While the cursor may have helped participants identify such central axis, the motion may not be intuitive.

Posture 1 (elbow rested high) is unique in that shoulder medial/lateral rotation and elbow flexion control vertical and horizontal movements, respectively, while in the other postures it is the inverse mapping. In addition, the physical input space is much larger, as participants had access to the lower hemisphere. Curiously, this mapping was beneficial for targets in the North and West zones. This finding strengthens the central axis hypothesis (aforementioned), as in elbow rested high the horizontal range is mostly symmetrical. In fact, in this posture nearly the entire ranges for elbow flexion and shoulder medial/lateral rotation are available, which may explain the increased physical demand that participants reported.

\section{GENERAL DISCUSSION}

\subsection{Further Design Considerations}

One design consideration revolves around scaling the motor movement to larger or small displays. For example, if needing to interact with a large wall display, Elbow-Anchored motions would still support such environments without any changes to the mapping function form-the output dimensions are just a parameter of the mapping. The same applies to when we need to scale down to interacting with a smaller display, such as in an AutoUI. We are not advocating for the replacement of remote-controlled devices. Instead, Elbow-Anchored input, as other mid-air interactions, offers a 

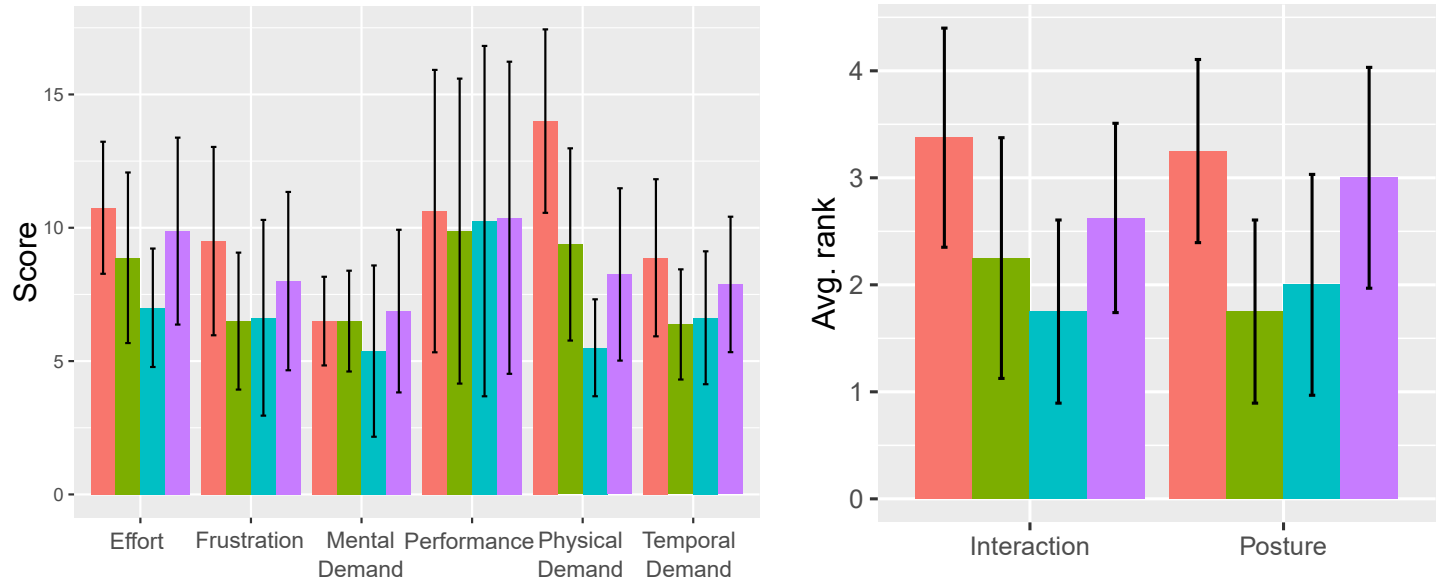

Posture

Elbow Rested High

Elbow Rested Low

Sideways Seated

Sideways Lying

Figure 15: Responses to questionnaires. On the left, Raw NASA TLX administered after each posture was completed. On the right, a preference ranking (lower is better) for postures and for mid-air interaction in each posture (based on experience in our user study).

viable alternative to the solution of seeking a lost remote, or having to sequentially interact with an interface, such as with a directional pad (DPAD). Future design iterations of our proposed layouts will be necessary to ensure cross compatibility with both restful and device-based interactions. Furthermore, additional consideration is needed to intelligently assume the user's posture and provide an interactive model without needing to calibrate for each posture. One approach might be to use the available cameras on such displays to interpret the posture and adjust the input model accordingly.

\subsection{Applications}

A number of applications, aside from our demonstration on SmartTV input, can benefit from Elbow-Anchored interactions. For example, mid-air text-entry keyboards [32] can be mapped to the movements we demonstrate. Our throughput results indicate regions to be avoided for placing characters, and instead could be used for presenting text or other feedback. While we currently use mouse click for selecting an item, when designed to operate using a smartwatch, additional approaches such as pinching the thumb and index [17] could instead be leveraged to make input efficient.

As mid-air interactions gain prominence in Automotive UIs, we can imagine interacting with such systems using Elbow-Anchored input. In many cases, with the presence of an arm-rest, the user's elbow has an available support for enabling comfortable and potentially less fatiguing input, than if the arm was held in mid-air. However, for such UIs, additional consideration is needed for providing suitable feedback, such as relying on audio or mid-air haptics.

Finally, emerging AR/VR platforms could benefit from such midair interaction capabilities, provided that a method for resolving depth ambiguity is applied, given that our mapping only supports 2D output spaces. Many video games are played in VR while sitting. As such, an available arm rest could be employed for lengthy interactions. We note from our results that there is no loss of performance in comparison to a shoulder-based mid-air input allowing gamers to reap the benefits from using an anchored elbow during long gaming sessions.

\subsection{Limitations}

Our results were derived from mostly right-handed participants, and additional work is required to ensure that Elbow-Anchored input is agnostic to hand-specific operations. For the majority of cases, we can assume that range-of-motion, shoulder medial/lateral rotation and elbow flexion-extension, operate in a mirrored and symmetrical fashion [40]. Additional work is needed to ensure that all users can benefit from Elbow-Anchored input. We demonstrate that elbow-anchored input is possible under a variety of postures. However, such postures are not exhaustive. Additionally, as there was no significant performance difference between between the postures, these minor differences may not warrant a redesign of the input space or the UI. However, for VR interactions, it may be necessary to research for ways to mitigate the inefficiencies we found, as VR users would likely interact with systems for a longer period of time and with smaller targets.Furthermore, we use the couch as it constitutes a central furnishing in homes (in most cultures), however, elbow-anchored should also be examined for postures when seated on the floor or in alternative arrangements. Finally, the spherical mapping enables input using a self-contained system, such as when the user is wearing a smartwatch. However, additional work is needed to decouple the device from the user, and instead use more generic capture methods, such as cameras in cars or on smartTVs, to understand the user's postures and apply our input model accordingly.

\section{CONCLUSION}

Posture is an important factor in user interaction at home. Users adopt a myriad of postures in the couch, and we have shown that posture influences which arm and hand motions are activated in common mid-air interaction, which in turn may affect performance and comfort. The availability of resting surfaces in common home furniture constitutes an opportunity for restful interaction, as many users' preferred posture involve a rested elbow. Traditional visionbased, planar input spaces, do not adapt well to users' postures. Instead, users are required to reposition to make their hand visible 
to the camera, and lift their elbow to accomplish axis aligned hand movements. We have shown that an elbow-anchored spherical input space has overall performance equivalent to that of planar input spaces, whether planar input spaces are anchored at the elbow or not. However, we have identified weak spots within spherical spaces that are related to the non-linearity of the mapping and motor constraints. Spherical input spaces defined relative to the body "follow" the user regardless of posture, and can be leveraged for posture-agnostic interaction. We have shown that, in practice, such spherical input spaces are relatively stable across postures, but that the spatial weak spots vary as a function of posture. We suggest such variations are only important in applications where the user is expected to interact for long durations and where target size cannot be easily controlled.

\section{REFERENCES}

[1] 2014. Input Methods / SAMSUNG Developers. Retrieved September 03, 2019 from https://developer.samsung.com/tv/develop/legacy-platform-library/2014/ 03_input_method

[2] 2017. This Car Gadget Can Help You Keep Your Eyes On the Road. Retrieved January 08, 2020 from https://time.com/4583451/navdy-heads-up-display/

[3] 2019. LG G8 ThinQ air motion: Learn how touchless works. Retrieved September 03, 2019 from https://lg.com/us/mobile-phones/g8-thinq/air-motion

[4] 2019. Thumbs Up: Hand Tracking on Oculus Quest This Week. Retrieved January 08, 2020 from https://www.oculus.com/blog/thumbs-up-hand-tracking-nowavailable-on-oculus-quest/?locale=en_US

[5] 2020. Control your Pixel without touching it. Retrieved January 08, 2020 from https://support.google.com/pixelphone/answer/9517454?hl=en

[6] Ilhan Aslan, Tabea Schmidt, Jens Woehrle, Lukas Vogel, and Elisabeth André 2018. Pen + Mid-Air Gestures: Eliciting Contextual Gestures. In Proceedings of the 20th ACM International Conference on Multimodal Interaction (Boulder, CO, USA) (ICMI '18). Association for Computing Machinery, New York, NY, USA, 135-144. https://doi.org/10.1145/3242969.3242979

[7] Myroslav Bachynskyi, Gregorio Palmas, Antti Oulasvirta, Jürgen Steimle, and Tino Weinkauf. 2015. Performance and Ergonomics of Touch Surfaces: A Comparative Study using Biomechanical Simulation. In Proceedings of the SIGCHI Conference on Human Factors in Computing Systems (Seoul, Korea) (CHI '15) ACM, New York, NY, USA, 10 pages.

[8] Gilles Bailly, Robert Walter, Jörg Müller, Tongyan Ning, and Eric Lecolinet. 2011 Comparing free hand menu techniques for distant displays using linear, marking and finger-count menus. In IFIP Conference on Human-Computer Interaction. Springer, 248-262.

[9] Renaud Blanch, Yves Guiard, and Michel Beaudouin-Lafon. 2004. Semantic Pointing: Improving Target Acquisition with Control-Display Ratio Adaptation. In Proceedings of the SIGCHI Conference on Human Factors in Computing Systems (Vienna, Austria) (CHI '04). Association for Computing Machinery, New York, NY, USA, 519-526. https://doi.org/10.1145/985692.985758

[10] Michelle A. Brown, Wolfgang Stuerzlinger, and E. J. Mendonça Filho. 2014. The Performance of Un-Instrumented in-Air Pointing. In Proceedings of Graphics Interface 2014 (Montreal, Quebec, Canada) (GI '14). Canadian Information Processing Society, CAN, 59-66.

[11] Debaleena Chattopadhyay and Davide Bolchini. 2014. Touchless circular menus: toward an intuitive UI for touchless interactions with large displays. In Proceedings of the International Working Conference on Advanced Visual Interfaces. ACM, Association for Computing Machinery, New York, NY, USA, 33-40.

[12] Debaleena Chattopadhyay and Davide Bolchini. 2015. Motor-intuitive interactions based on image schemas: Aligning touchless interaction primitives with human sensorimotor abilities. Interacting with Computers 27, 3 (2015), 327-343.

[13] Andy Cockburn and Andrew Firth. 2003. Improving the Acquisition of Small Targets. People and Computers (04 2003). https://doi.org/10.1007/978-1-44713754-2_11

[14] Andy Cockburn, Philip Quinn, Carl Gutwin, Gonzalo Ramos, and Julian Looser. 2011. Air pointing: Design and evaluation of spatial target acquisition with and without visual feedback. International fournal of Human-Computer Studies 69, 6 (2011), 401-414.

[15] Clifton Forlines, Daniel Vogel, and Ravin Balakrishnan. 2006. HybridPointing Fluid Switching between Absolute and Relative Pointing with a Direct Input Device. In Proceedings of the 19th Annual ACM Symposium on User Interface Software and Technology (Montreux, Switzerland) (UIST '06). Association for Computing Machinery, New York, NY, USA, 211-220. https://doi.org/10.1145/ 1166253.1166286

[16] Dustin Freeman, Ramadevi Vennelakanti, and Sriganesh Madhvanath. 2012. Freehand pose-based gestural interaction: Studies and implications for interface design. In Proceedings of the 4th International Conference on Intelligent Human Computer Interaction (IHCI). IEEE, 1-6.

[17] Jun Gong, Xing-Dong Yang, and Pourang Irani. 2016. Wristwhirl: One-handed continuous smartwatch input using wrist gestures. In Proceedings of the 29th Annual Symposium on User Interface Software and Technology. Association for Computing Machinery, New York, NY, USA, 861-872.

[18] François Guimbretière and Chau Nguyen. 2012. Bimanual marking menu for near surface interactions. In Proceedings of the SIGCHI conference on human factors in computing systems. ACM, 825-828.

[19] Darren Guinness, Alvin Jude, G Michael Poor, and Ashley Dover. 2015. Models for rested touchless gestural interaction. In Proceedings of the 3rd ACM Symposium on Spatial User Interaction. ACM, 34-43.

[20] Sandra G Hart. 2006. NASA-task load index (NASA-TLX); 20 years later. In Proceedings of the human factors and ergonomics society annual meeting, Vol. 50. Sage publications Sage CA: Los Angeles, CA, 904-908.

[21] Juan David Hincapié-Ramos, Xiang Guo, Paymahn Moghadasian, and Pourang Irani. 2014. Consumed endurance: a metric to quantify arm fatigue of midair interactions. In Proceedings of the SIGCHI Conference on Human Factors in Computing Systems. ACM, Association for Computing Machinery, New York, NY, USA, 1063-1072.

[22] Sujin Jang, Wolfgang Stuerzlinger, Satyajit Ambike, and Karthik Ramani. 2017. Modeling Cumulative Arm Fatigue in Mid-Air Interaction Based on Perceived Exertion and Kinetics of Arm Motion. In Proceedings of the 2017 CHI Conference on Human Factors in Computing Systems (Denver, Colorado, USA) (CHI '17). Association for Computing Machinery, New York, NY, USA, 3328-3339. https: //doi.org/10.1145/3025453.3025523

[23] Alvin Jude, G. Michael Poor, and Darren Guinness. 2014. Personal Space: User Defined Gesture Space for GUI Interaction. In CHI '14 Extended Abstracts on Human Factors in Computing Systems (Toronto, Ontario, Canada) (CHI EA '14). Association for Computing Machinery, New York, NY, USA, 1615-1620. https: //doi.org/10.1145/2559206.2581242

[24] Daniel Kharlamov, Brandon Woodard, Liudmila Tahai, and Krzysztof Pietroszek. 2016. TickTockRay: Smartwatch-Based 3D Pointing for Smartphone-Based Virtual Reality. In Proceedings of the 22nd ACM Conference on Virtual Reality Software and Technology (Munich, Germany) (VRST '16). Association for Computing Machinery, New York, NY, USA, 365-366. https://doi.org/10.1145/2993369.2996311

[25] Michael Kipp. 2008. Spatiotemporal Coding in ANVIL. In Proceedings of the Sixth International Conference on Language Resources and Evaluation (LREC'08).

[26] Werner A. König, Jens Gerken, Stefan Dierdorf, and Harald Reiterer. 2009. Adaptive Pointing: Implicit Gain Adaptation for Absolute Pointing Devices. In $\mathrm{CHI}$ '09 Extended Abstracts on Human Factors in Computing Systems (Boston, MA, USA) (CHI EA '09). Association for Computing Machinery, New York, NY, USA, 4171-4176. https://doi.org/10.1145/1520340.1520635

[27] Arun Kulshreshth and Joseph J LaViola Jr. 2014. Exploring the usefulness of finger-based 3D gesture menu selection. In Proceedings of the SIGCHI Conference on Human Factors in Computing Systems. ACM, Association for Computing Machinery, New York, NY, USA, 1093-1102.

[28] Frank Chun Yat Li, David Dearman, and Khai N Truong. 2009. Virtual shelves: interactions with orientation aware devices. In Proceedings of the 22nd Annual ACM Symposium on User Interface Software and Technology. Association for Computing Machinery, New York, NY, USA, 125-128.

[29] Jing Z. Liu, Robert W. Brown, and Guang H. Yue. 2002. A Dynamical Model of Muscle Activation, Fatigue, and Recovery. Biophysical fournal 82, 5 (2002), 2344 - 2359. https://doi.org/10.1016/S0006-3495(02)75580-X

[30] Mingyu Liu, Mathieu Nancel, and Daniel Vogel. 2015. Gunslinger: Subtle Armsdown Mid-Air Interaction. In Proceedings of the 28th Annual ACM Symposium on User Interface Software \& Technology (Charlotte, NC, USA) (UIST '15). Association for Computing Machinery, New York, NY, USA, 63-71. https://doi.org/10.1145/ 2807442.2807489

[31] Paul Lubos, Gerd Bruder, Oscar Ariza, and Frank Steinicke. 2016. Touching the sphere: Leveraging joint-centered kinespheres for spatial user interaction. In Proceedings of the 2016 Symposium on Spatial User Interaction. Association for Computing Machinery, New York, NY, USA, 13-22.

[32] Anders Markussen, Mikkel Rønne Jakobsen, and Kasper Hornbæk. 2014. Vulture: a mid-air word-gesture keyboard. In Proceedings of the SIGCHI Conference on Human Factors in Computing Systems. Association for Computing Machinery, New York, NY, USA, 1073-1082.

[33] David E. Meyer, Richard A. Abrams, Sylvan Kornblum, Charles E. Wright, and J. E. Keith Smith. 1988. Optimality in human motor performance: ideal control of rapid aimed movements. Psychological Review 95 (1988), 340-370.

[34] Roberto A Montano Murillo, Sriram Subramanian, and Diego Martinez Plasencia. 2017. Erg-O: ergonomic optimization of immersive virtual environments. In Proceedings of the 30th Annual ACM Symposium on User Interface Software and Technology. Association for Computing Machinery, New York, NY, USA, 759-771.

[35] Richard Morey. 2008. Confidence Intervals from Normalized Data: A correction to Cousineau (2005). Tutorials in Quantitative Methods for Psychology 4 (09 2008). https://doi.org/10.20982/tqmp.04.2.p061 
[36] Meredith Ringel Morris, Andreea Danielescu, Steven Drucker, Danyel Fisher, Bongshin Lee, m. c. schraefel, and Jacob O. Wobbrock. 2014. Reducing Legacy Bias in Gesture Elicitation Studies. Interactions 21, 3 (May 2014), 40-45. https //doi.org/10.1145/2591689

[37] Brad A. Myers, Rishi Bhatnagar, Jeffrey Nichols, Choon Hong Peck, Dave Kong, Robert Miller, and A. Chris Long. 2002. Interacting at a Distance: Measuring the Performance of Laser Pointers and Other Devices. In Proceedings of the SIGCH Conference on Human Factors in Computing Systems (Minneapolis, Minnesota, USA) (CHI '02). Association for Computing Machinery, New York, NY, USA, 33-40. https://doi.org/10.1145/503376.503383

[38] Mathieu Nancel, Emmanuel Pietriga, Olivier Chapuis, and Michel BeaudouinLafon. 2015. Mid-Air Pointing on Ultra-Walls. ACM Trans. Comput.-Hum. Interact. 22, 5, Article 21 (Aug. 2015), 62 pages. https://doi.org/10.1145/2766448

[39] Mathieu Nancel, Julie Wagner, Emmanuel Pietriga, Olivier Chapuis, and Wendy Mackay. 2011. Mid-Air Pan-and-Zoom on Wall-Sized Displays. In Proceedings of the SIGCHI Conference on Human Factors in Computing Systems (Vancouver, BC, Canada) (CHI '11). Association for Computing Machinery, New York, NY, USA 177-186. https://doi.org/10.1145/1978942.1978969

[40] J NASA. 2000. Anthropometry and biomechanics. Man systems integration standards 1 (2000)

[41] Fabrizio Nunnari, Myroslav Bachynskyi, and Alexis Heloir. 2016. Introducing postural variability improves the distribution of muscular loads during mid-air gestural interaction. In Proceedings of the 9th International Conference on Motion in Games. ACM, Association for Computing Machinery, New York, NY, USA
$155-160$.

[42] Ji-Young Oh and Wolfgang Stuerzlinger. 2002. Laser Pointers as Collaborative Pointing Devices. Proceedings - Graphics Interface 2002 (10 2002).

[43] Jose Pinheiro, Douglas Bates, Saikat DebRoy, Deepayan Sarkar, and R Core Team. 2020. nlme: Linear and Nonlinear Mixed Effects Models. https://CRAN.Rproject.org/package=nlme $\mathrm{R}$ package version 3.1-147.

[44] Jakub Segen and Senthil Kumar. 2000. Look Ma, No Mouse! Commun. ACM 43, 7 (July 2000), 102-109. https://doi.org/10.1145/341852.341869

[45] David E Vaillancourt and Karl M Newell. 2000. Amplitude changes in the 8-12, $20-25$, and $40 \mathrm{~Hz}$ oscillations in finger tremor. Clinical Neurophysiology 111,10 (2000), 1792 - 1801. https://doi.org/10.1016/S1388-2457(00)00378-3

[46] Daniel Vogel and Ravin Balakrishnan. 2005. Distant freehand pointing and clicking on very large, high resolution displays. In Proceedings of the 18th annual ACM Symposium on User Interface Software and Technology. Association for Computing Machinery, New York, NY, USA, 33-42.

[47] Johann Wentzel, Greg d'Eon, and Daniel Vogel. 2020. Improving Virtual Reality Ergonomics through Reach-Bounded Non-Linear Input Amplification. In Proceedings of the 2020 CHI Conference on Human Factors in Computing Systems (Honolulu, HI USA) (CHI '20). Association for Computing Machinery, New York, NY, USA, Article 558, 12 pages. https://doi.org/10.1145/3313831.3376687

[48] Ting Xia and Laura Law. 2008. A theoretical approach for modeling peripheral muscle fatigue and recovery. Fournal of biomechanics 41 (10 2008), 3046-52. https://doi.org/10.1016/j.jbiomech.2008.07.013 